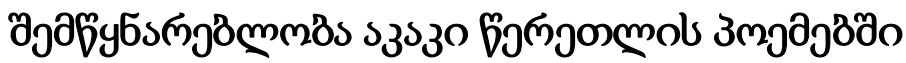

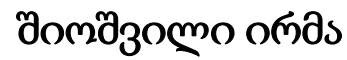

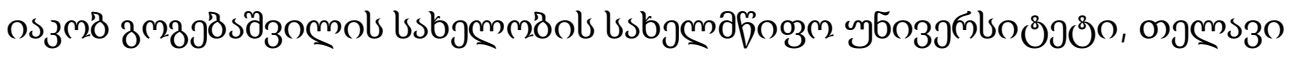

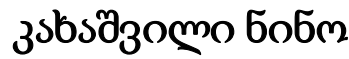

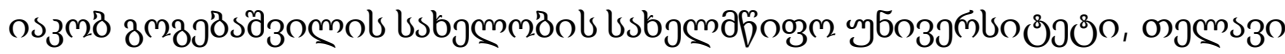 \\ https://doi.org/10.52340/idw.2021.524
}

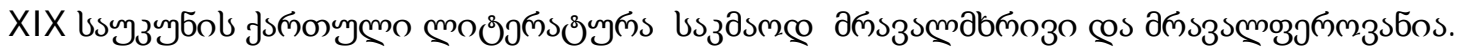

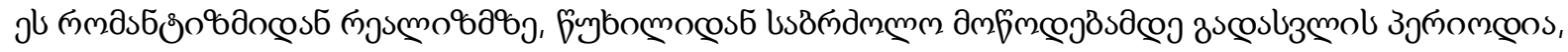

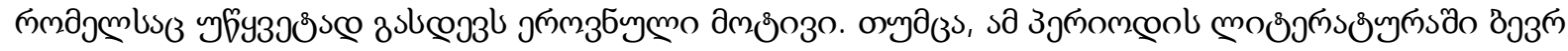

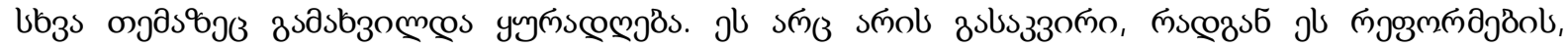

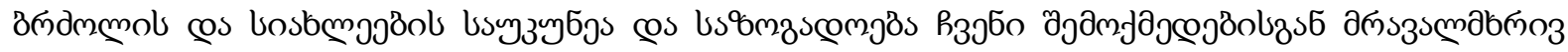

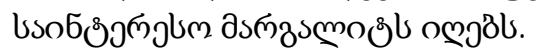

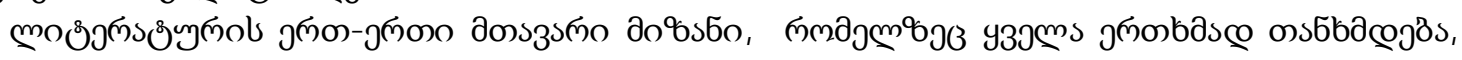

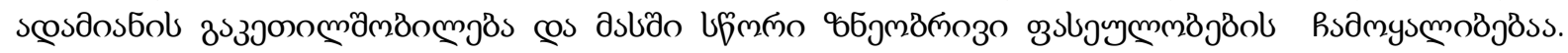

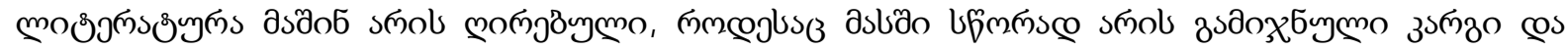

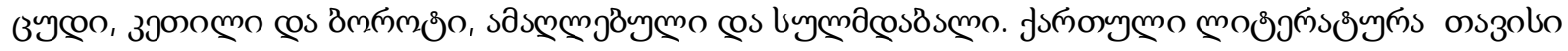

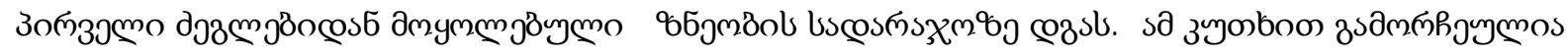

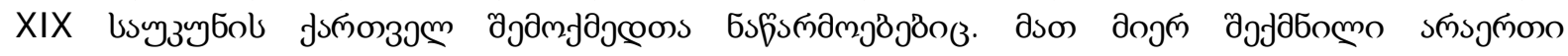

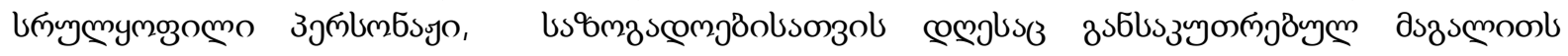

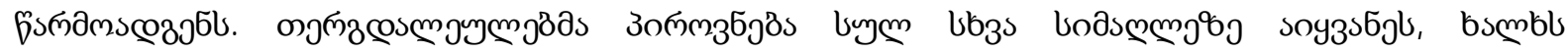

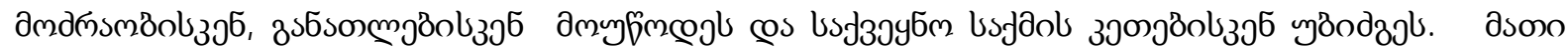

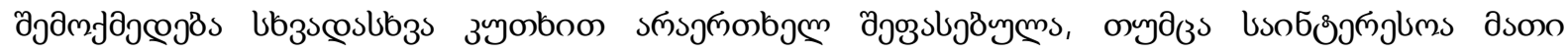

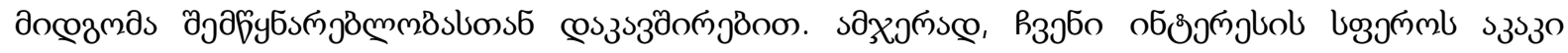

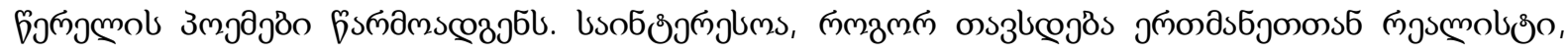

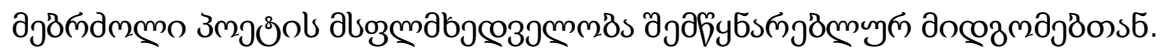

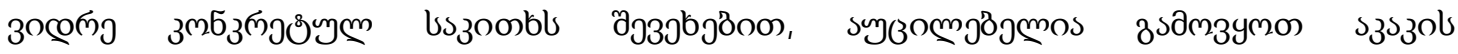

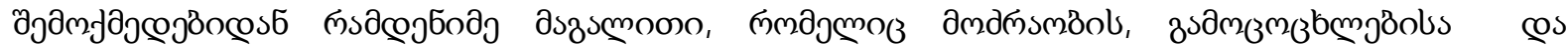

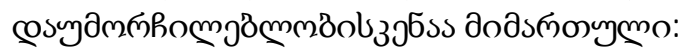

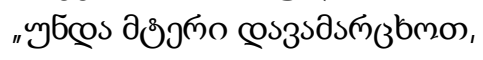

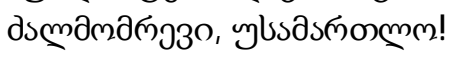

ju оңб

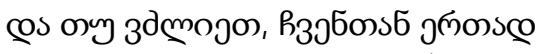

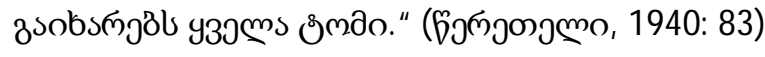

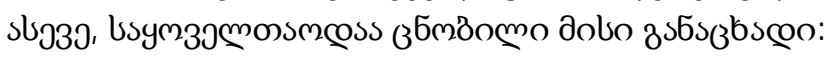

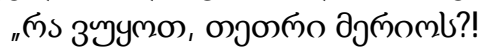

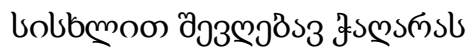

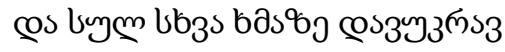

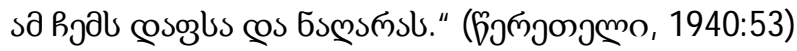
1940:72)

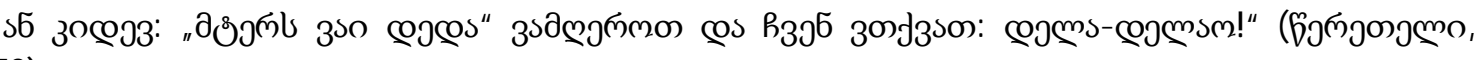

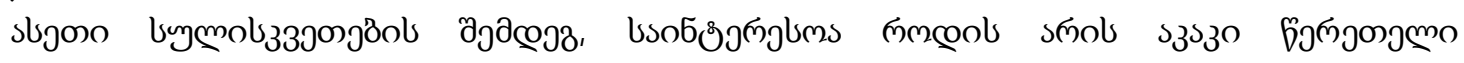




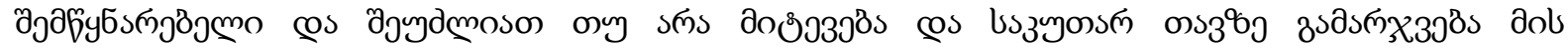

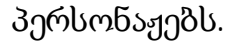

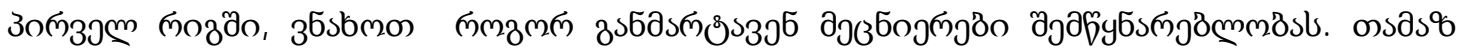

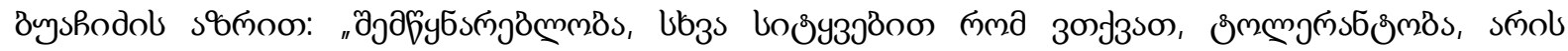

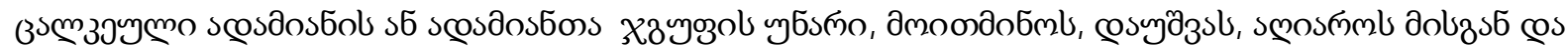

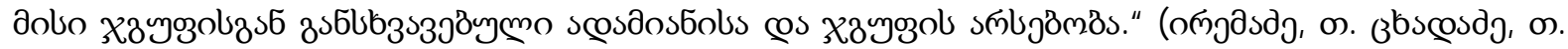

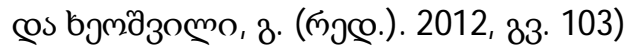

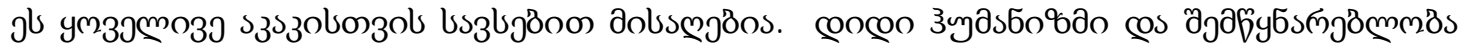

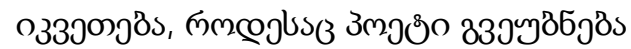

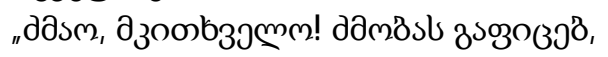

з

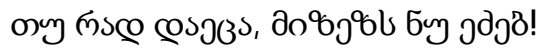

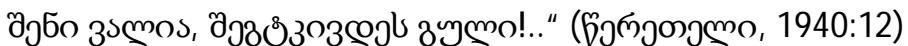

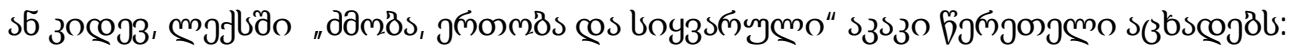

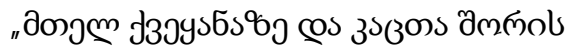

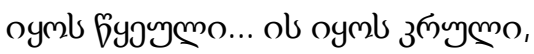

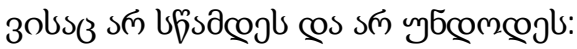

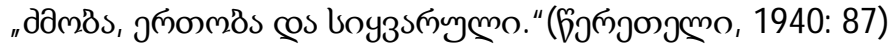

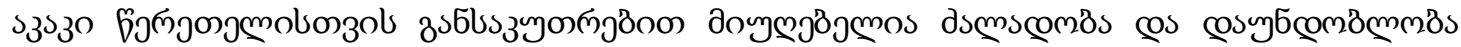
dasons Әмко

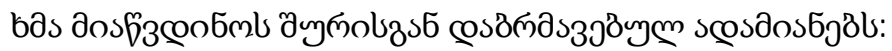

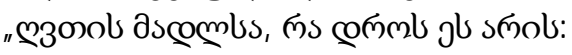

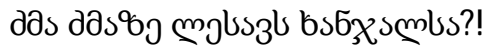

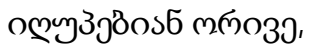

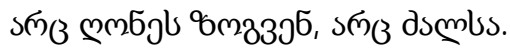

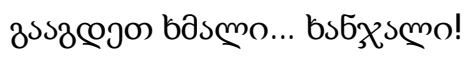

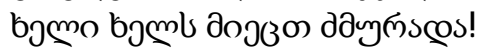

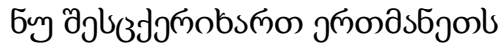

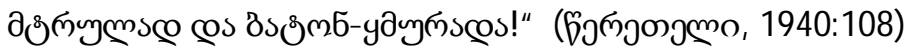

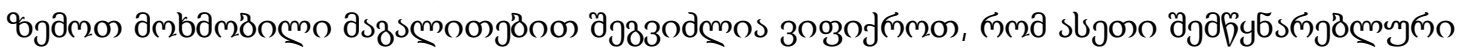

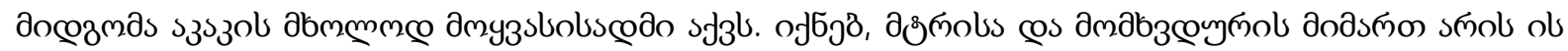

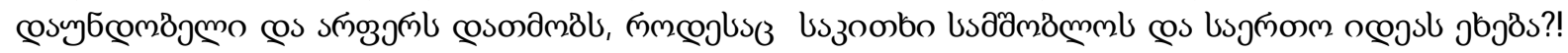

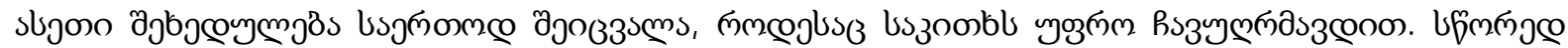

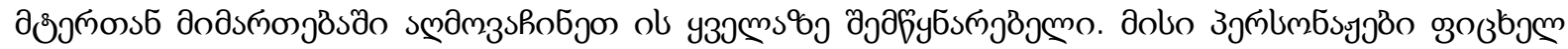

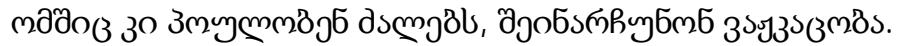

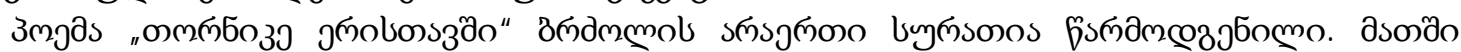

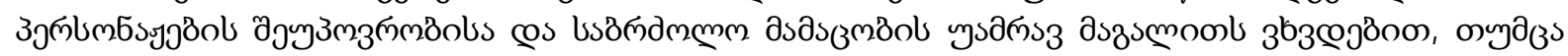

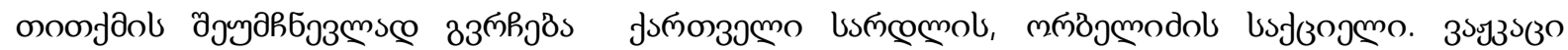

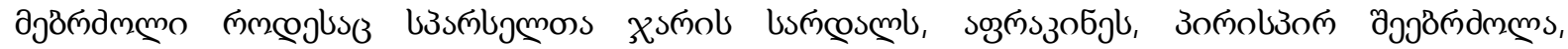

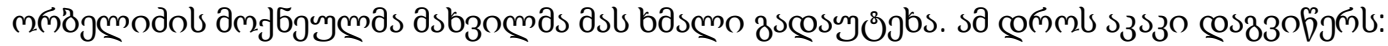

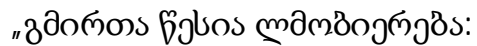

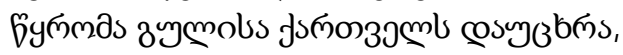

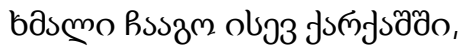

Rsamjosms cos sly yonbms:

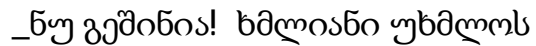

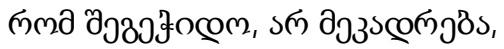

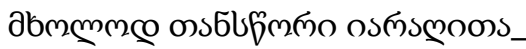

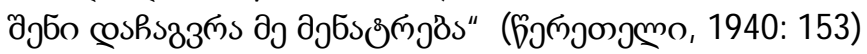

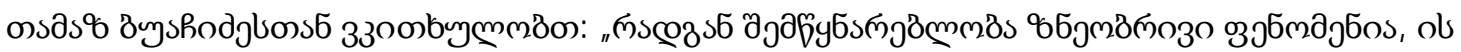




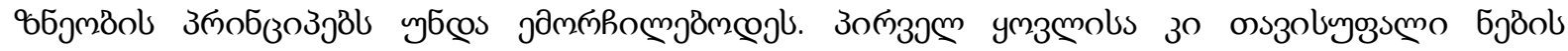

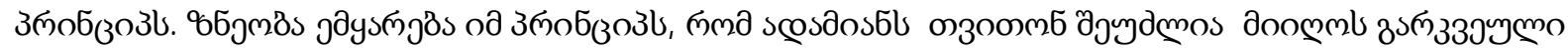

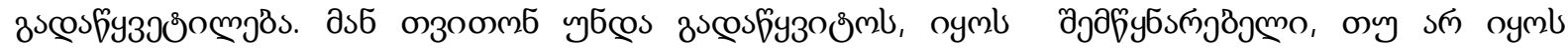

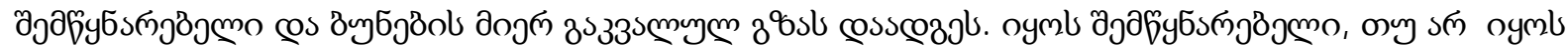

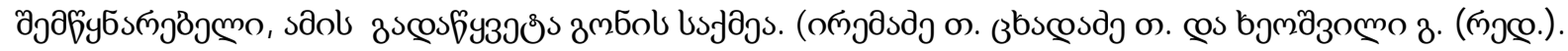
2012: 105)

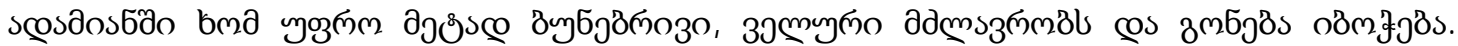

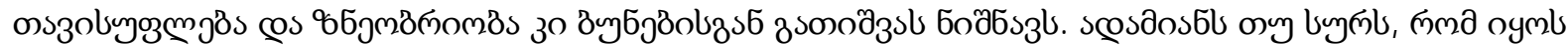

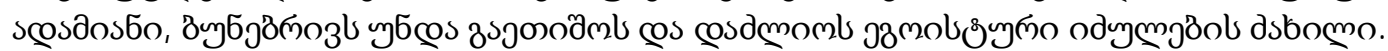

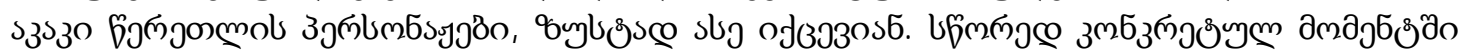

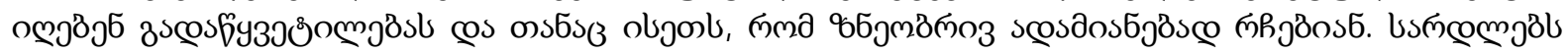

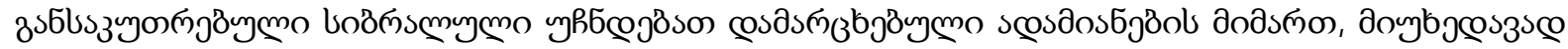

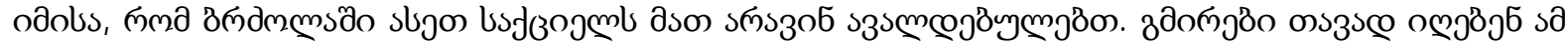

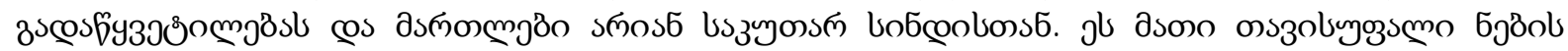

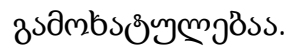

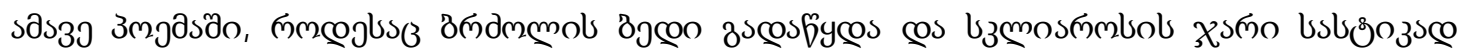

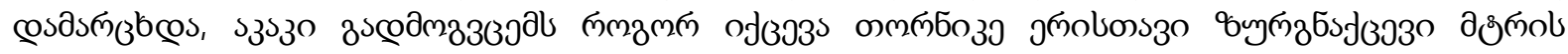

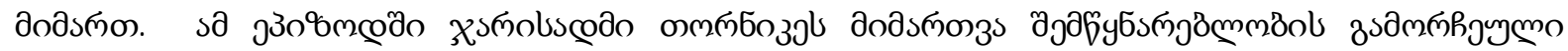
азzsмnoonos:

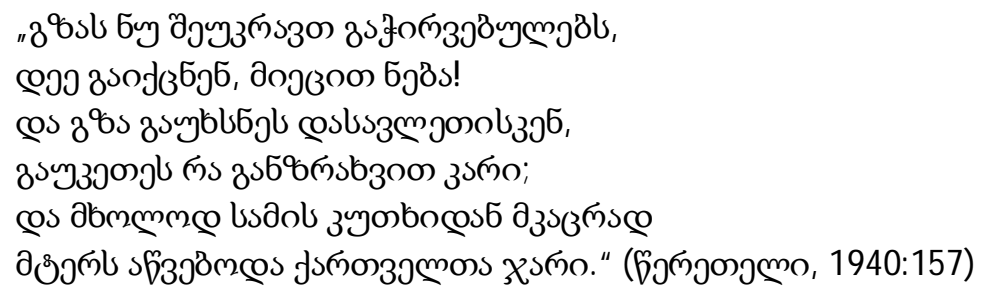

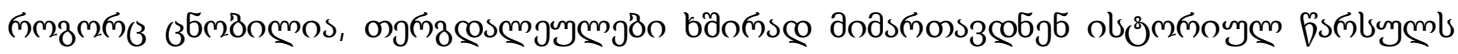

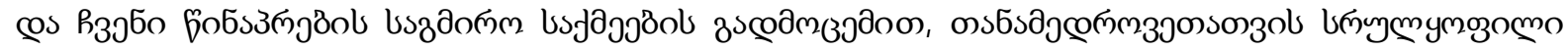

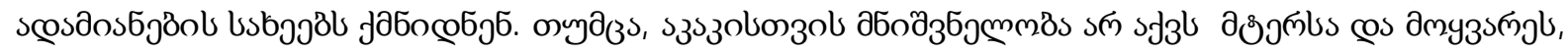

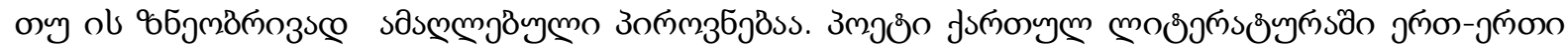

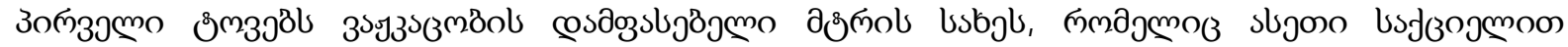

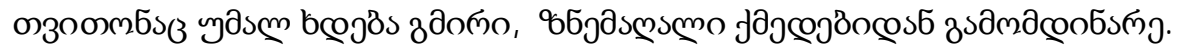

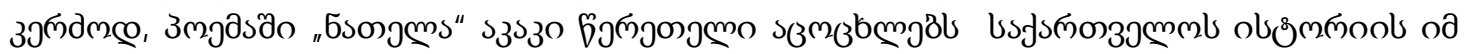

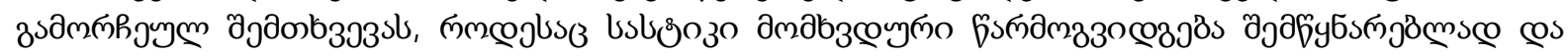

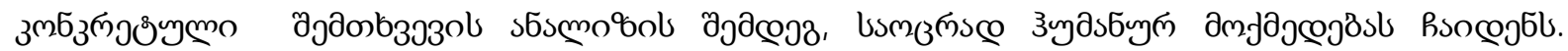

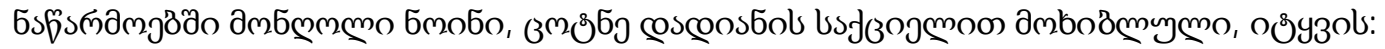

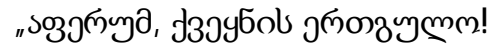

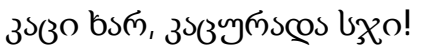

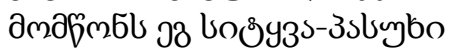

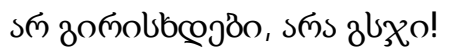

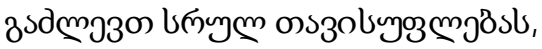

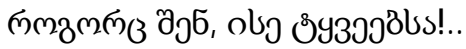

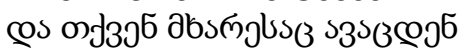

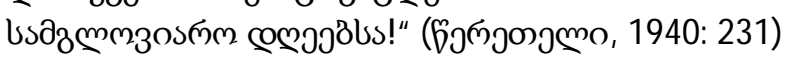

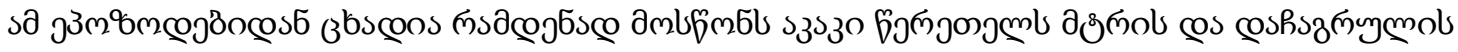

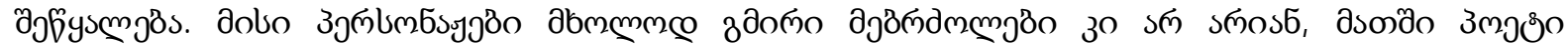

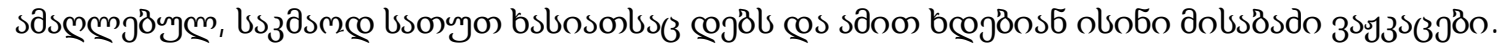

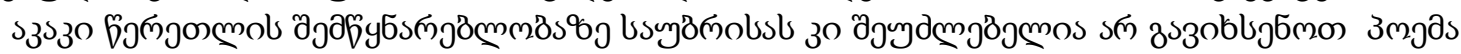

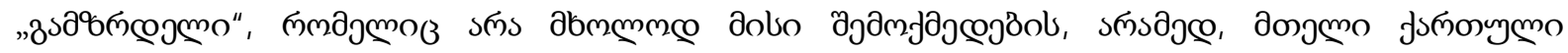

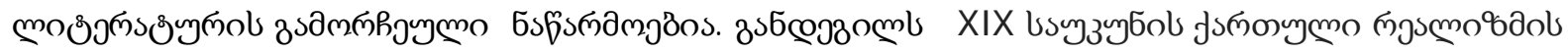




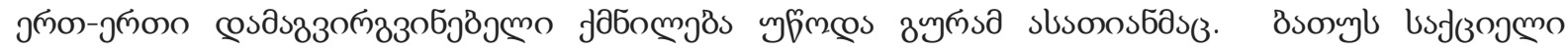

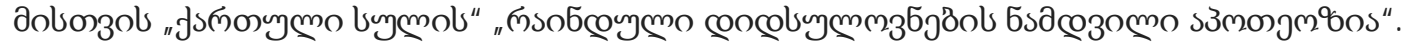

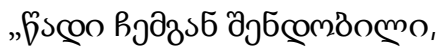

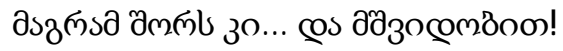

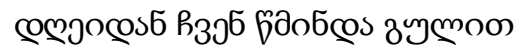

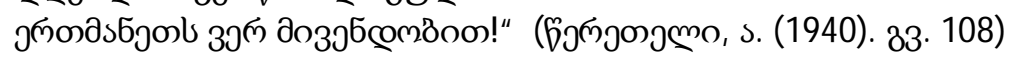

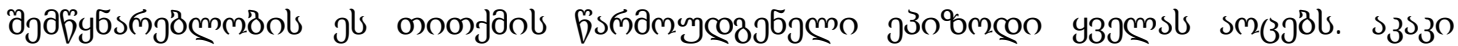

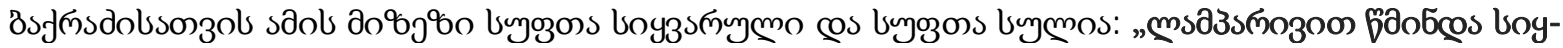

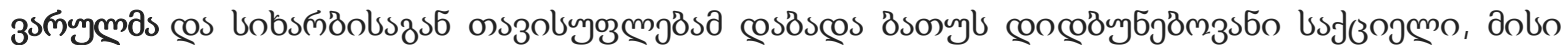

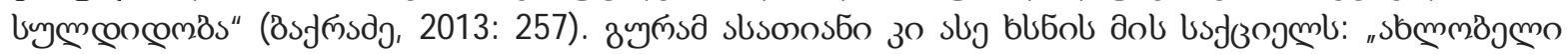

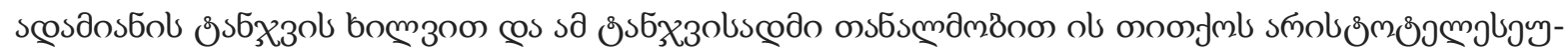

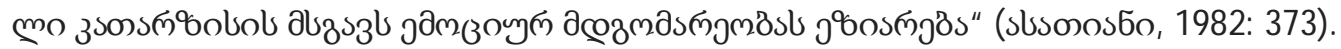

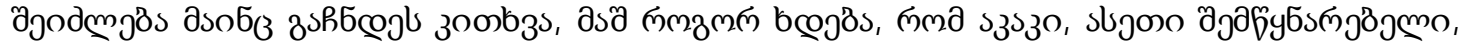

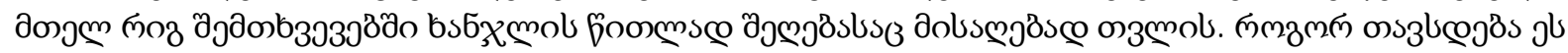

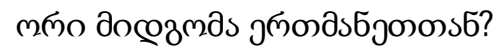

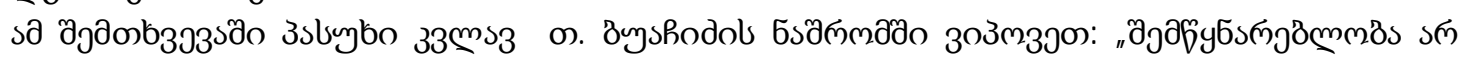

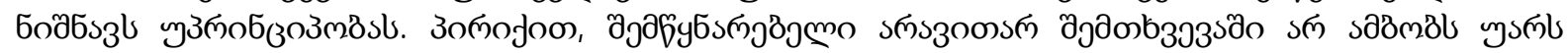

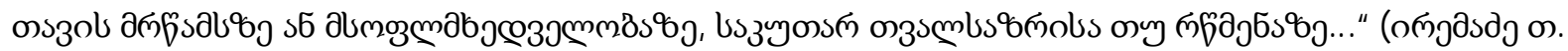

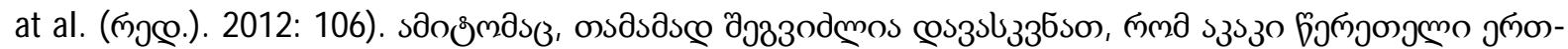

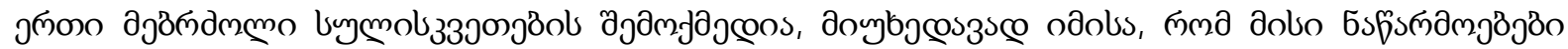

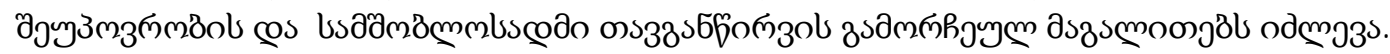

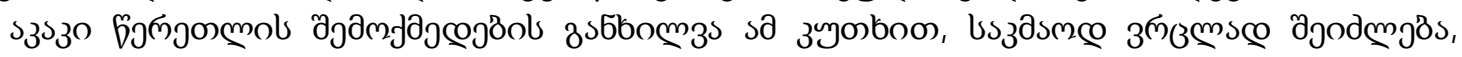

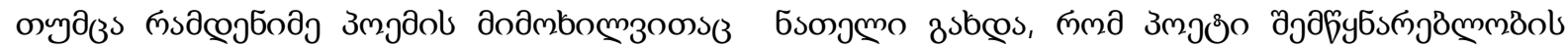

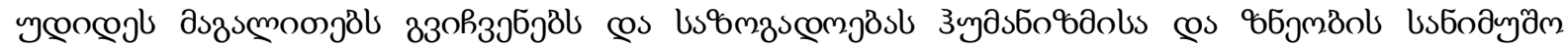

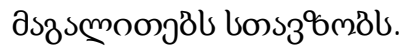

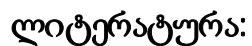

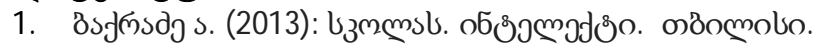

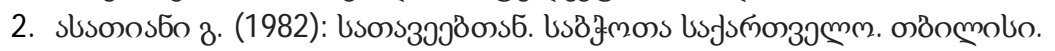

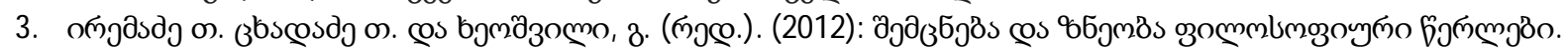

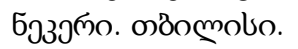

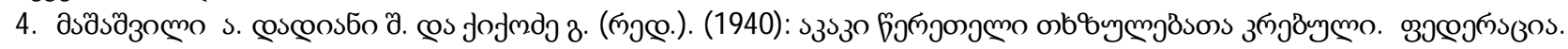
๓в̈omolo.

\section{Tolerance in Akaki Tsereteli’s Poems}

\author{
Shioshvili Irma \\ Iakob Gogebashvili State Universiti, Telavi \\ Kakhashvili Nino \\ Iakob Gogebashvili State Universiti, Telavi
}

\begin{abstract}
XIX century Georgian literature is quite versatile and diverse. It is the transitional period from Romanticism to Realism that is continuously accompanied by the national motive. Though, many other topics were also emphasized in literature of the mentioned period.

Georgian literature beginning with its first works guards the morality. Works of XIX century Georgian creators are distinguished in that regard as well. Different ideal characters created by them are the special examples for society even today. This time the given work deals with Akaki Tsereteli's poems. It is worth of interest as how the ideology of realist, warrior poet is compatible
\end{abstract}


with the tolerant approaches, when Akaki Tsereteli is tolerant and if their characters can forgive and gain a victory over themselves.

Tolerance as the moral phenomenon and its philosophical definition are also emphasized in the given work in an interesting way. All of the abovementioned matters are quite acceptable for Akaki. Great humanism and tolerance are revealed in poet's words.

After analyzing the poems given in the work it becomes clear that the poet is tolerant not only with neighbors, but also he turns out to be the most gracious one with enemies. His characters find ways for showing courage even in the war. His characters are not only heroic warriors, the poet also gives them quite a tender temper and that is the reason for becoming worthy brave man.

His characters are separated from the natural things, defeat selfish forces and make such decisions in a specific moment that helps them remain humans with morality, they are right with their own conscience and it is the expression of their free will.

The given work allows to conclude that Akaki Tsereteli's poems reveal the greatest examples of tolerance and offer society the samples of humanism and morality.

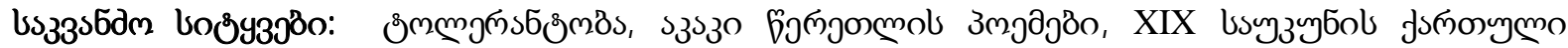

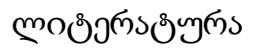

Key words: Tolerance, Akaki Tsereteli's Poems, XIX century Georgian literature. 